\title{
Benchmark RGB-D Gait Datasets: A Systematic Review
}

\author{
João Ferreira Nunes ${ }^{1,2[0000-0002-5204-4043]}$, \\ Pedro Miguel Moreira ${ }^{1[0000-0001-8371-0347]}$, and \\ João Manuel R. S. Tavares 2[0000-0001-7603-6526] \\ 1 ARC4DigiT - Centro de Investigação Aplicada para a Transformação Digital, \\ Instituto Politécnico de Viana do Castelo, PORTUGAL \\ \{joao.nunes, pmoreira\}@estg.ipvc.pt \\ 2 Instituto de Ciência e Inovação em Engenharia Mecânica e Engenharia Industrial, \\ Departamento de Engenharia Mecânica, Faculdade de Enhenharia, Universidade do \\ Porto, Porto, PORTUGAL \\ tavares@fe.up.pt
}

\begin{abstract}
Human motion analysis has proven to be a great source of information for a wide range of applications. Several approaches for a detailed and accurate motion analysis have been proposed in the literature, as well as an almost proportional number of dedicated datasets. The relatively recent arrival of depth sensors contributed to an increasing interest in this research area and also to the emergence of a new type of motion datasets. This work focuses on a systematic review of publicly available depth-based datasets, encompassing human gait data which is used for person recognition and/or classification purposes. We have conducted this systematic review using the Scopus database. The herein presented survey, which to the best of our knowledge is the first one dedicated to this type of datasets, is intended to inform and aid researchers on the selection of the most suitable datasets to develop, test and compare their algorithms.
\end{abstract}

Keywords: Gait Datasets · Depth Sensors - Systematic Review.

\section{Introduction}

The analysis of human motions has been a very active research topic, with a manifold of potential applications. Some of them have already been discussed in reviews like 22 27/2030. Particularly, gait, as a specific type of human movement, has been used for a wide range of applications, either as a data source or simply as a way to interact with them. For example, in healthcare, gait data has been used i) for geriatrics assistance (elderly fall prevention [15]19, mobility assessment, and home monitoring [8]); ii) to conduct orthopedic studies [4]28; and iii) to neurological and chronic disorders assessment [9|12 32]). In the sports domain, gait patterns have been used to assist athletes so they can perform better and safer [26[13]). In smart surveillance systems, gait signatures are used 
as a new type of biometric authentication [6]7]). And also, in human-computer interaction, where gait has been used to interact in video-game environments [3] or to animate virtual characters [14].

Several methodologies for a detailed and accurate gait analysis have been proposed, including computer vision-based, inertial sensors-based or other based approaches. Simultaneously, dedicated gait datasets have appeared almost in a proportional way. The relatively recent arrival of depth sensors, like Microsoft Kinect, Intel RealSense or Asus Xtion, capable of tracking humans in real-time without the need to ware special suits or markers, contributed to an increasing interest in this research area leading also to the emergence of a new type of motion datasets. A detailed description of a widely used depth sensor functionality is given in 34. These depth-based datasets, besides the RGB data, also include raw depth data and in same cases also the $3 \mathrm{D}$ coordinates of a set of points that in general, correspond to human body joints.

Regardless the methodologies nor the technologies used, it is very important for the scientific community to use common input data, enabling coherent comparisons of performances and results. For that reason, and focusing specifically on the human gait, we have conducted this systematic review, whose primary goal is to identify all the existing, freely available, depth-based datasets containing human gait information, whose applicability encompasses person recognition and/or classification purposes. Consequently, we are assisting researchers by presenting an updated framework, easy to analyse, useful to identify existing datasets, and suitable to compare them, avoiding, eventually, the creation of new (and sometimes redundant) datasets.

\section{Related Work}

Several human motion-based datasets reviews can be found in the literature. Some examples are presented in $2421 / 7 / 29$. It is evident the diversity between datasets in terms of their applicability, the acquisition environment conditions, the number of participants, the number of sequences, etc. However, none of those reviews have been presented in a simple form, easy to analyse and to compare datasets. In most of the reviews each dataset is described without any formal organization or structure. Moreover, in the examples previously identified, the revised datasets do not include depth data. Nevertheless, in [10], it is presented a revision of depth-based datasets within eight categories: semantics, object pose estimation, camera tracking, scene reconstruction, object tracking, human actions, human faces and human identification. The latter encompasses four datasets, in which two of them are also present in our study.

\section{Systematic Review}

The protocol that was used to locate, gather and evaluate the datasets under study is described in this Section. The review took place in the first quarter of 2019 and it was conducted using the Scopus database. The criteria defined for the 
selection of articles were as follows: Domain (Gait Analysis); Purpose (Person Recognition and/or Gender Classification); and Dataset (Depth-Based).

Thus, the searched terms used in the Scopus database were: ("gait recognition" OR "gait identification" OR "person recognition" OR "person identification" OR "gender identification" OR "gender recognition") AND ("depth sensor" OR "RGBD" OR "RGB-D" OR "Kinect" OR "RealSense" OR "Xtion" OR "ToF") AND ("dataset" OR "database" OR "data set"). These terms were searched in the title, abstract and keywords of the indexed articles in Scopus database and were refined by: Publication types $=($ ALL $)$ AND Languages $=$ (ENGLISH). This search resulted in 58 articles.

Every resulting article was analysed and both self-constructed and referenced depth-based gait datasets were included in the first set of articles. From among the 58 articles retrieved, we were able to identify 10 freely available datasets, which are described in Section 4 A newly created dataset that was developed by us was also included in the list, thus resulting in a total of 11 datasets reviewed.

\section{Framework for Datasets Comparison}

For a simple and easy way to analyse and also to compare datasets we decided to present them in form of a table, describing each dataset based on a set of generic features. In this Section the features used to describe each dataset are listed, and then the selected datasets are identified. The proposed framework is presented in Table 1.

\subsection{Features Description}

The selected features were the following:

- applicability: the context for which the dataset was created;

- subjects: number of participants, and if possible, their description regarding gender and age;

- sensor: number and type of sensors used, and how they were placed on scene;

- trajectories: number of different defined trajectories;

- sequences/subject: number of sequences performed per subject;

- covariates: list of existing variations between sequences (e.g.: walking styles, clothing, etc.);

- collected data: list of data that was collected and made available to download;

- additional data supplied: list of additional data that was made accessible, like code/applications to manipulate data.

A few other features were initially included in the datasets description structure proposal, however, given that their values were constant between all datasets, we have decided to omit them. Those features included information about the environment where the sessions took place (indoor laboratories), about the frame rate of used sensors (30 fps) and about whether the participants were aware that they were being filmed (all participants were conscious that they were being observed). 
Selected Datasets The datasets that were part of our study, whose selection procedure was explained in Section 3, and that consequently are present in our proposed framework are the following: Depth-Based Gait Dataset [?], DGait [5, GRIDDS, 11, Kinect Gait Biometry Dataset 11, RGB-D Person Reidentification Dataset [2], SAIVT-DGD [25, SDUgait [31, SZU RGB-D Gait Dataset 33, TUM-GAID Database 16] and UPCV Gait Dataset [18.

\section{Conclusions}

The importance of human gait is quite evident, considering its wide range of application domains. Systems that are able to recognize humans and to classify human attributes like gender, age or mood may have a great impact in our society. In the last decade, the dissemination and availability of RGBD sensors (depth + image) prompted the development of new methods and the availability of datasets including depth information beyond the objects represented in the images. This work, to the best of our knowledge, is the first systematic review dedicated to depth-based gait datasets, whose purpose is person recognition and/or classification, covering a total of 11 datasets, of which $72 \%$ were acquired between 2011 and 2015, using the first version of the Kinect sensor, whilst 18\% were acquired between 2016 and 2018, using the second version of Kinect. Concerning the original context that they were created for, $81 \%$ aimed to recognize persons by their gait, while for the remaining $19 \%$ their goal was to classify people's gender. As an outcome of this work, we introduce our perspective on how these datasets can be classified and compared and a logical, structured and feature oriented classification framework is presented.

\section{References}

1. Andersson, V.O., Araújo, R.M.: Person identification using anthropometric and gait data from kinect sensor. In: Proceedings of the 29th Association for the Advancement of Artificial Intelligence. pp. 425-431 (2015)

2. Barbosa, I.B., Cristani, M., Bue, A.D., Bazzani, L., Murino, V.: Re-identification with RGB-d sensors. In: Proceedings of the 12th international conference on Computer Vision - Volume Part I. pp. 433-442. Springer Berlin Heidelberg (2012)

3. Bloom, V., Argyriou, V., Makris, D.: Hierarchical transfer learning for online recognition of compound actions. Computer Vision and Image Understanding 144, 62$72(2016)$

4. Bonnechère, B., Jansen, B., Salvia, P., Bouzahouene, H., Omelina, L., Moiseev, F., Sholukha, V., Cornelis, J., Rooze, M., Jan, S.V.S.: Validity and reliability of the kinect within functional assessment activities: Comparison with standard stereophotogrammetry. Gait \& Posture 39(1), 593-598 (2014)

5. Borràs, R., Lapedriza, À., Igual, L.: Depth information in human gait analysis: An experimental study on gender recognition. In: Proceedings of the 9th International Conference on Image Analysis and Recognition - Volume Part II. pp. 98-105. ICIAR, Springer Berlin Heidelberg (2012) 
Table 1. The proposed framework used to describe each dataset.

\begin{tabular}{|c|c|c|c|c|c|c|c|c|c|}
\hline 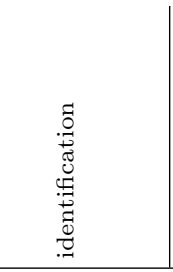 & 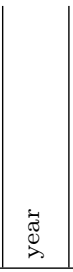 & 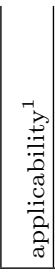 & $\begin{array}{l}\frac{n}{0} \\
0 \\
.0 \\
0 \\
0 \\
0 \\
0 \\
\# \\
\#\end{array}$ & $\begin{array}{l}\infty \\
\vdots \\
0 \\
0 \\
0 \\
0 \\
\# \\
\#\end{array}$ & 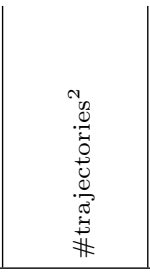 & 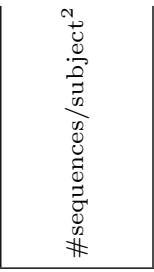 & 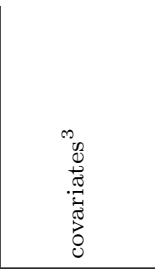 & 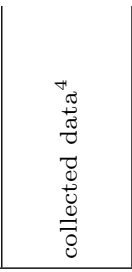 & 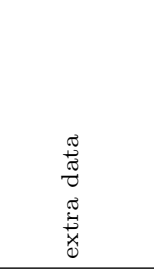 \\
\hline $\begin{array}{l}\text { BVH MoCap } \\
\text { Databse } \\
11\end{array}$ & 2010 & PR & \begin{tabular}{|l|}
$6+4$ \\
static + \\
moving \\
sessions
\end{tabular} & $\begin{array}{l}\text { Kinect v1 } \\
\text { static or } \\
\text { in motion }\end{array}$ & $\begin{array}{c}5 \\
(2 \mathrm{~S}+\mathrm{F}+ \\
\mathrm{B}+\mathrm{MS})\end{array}$ & IN & $\mathrm{SP}$ & SK & $\begin{array}{c}\text { c\# code }+ \\
\text { matlab } \\
\text { scripts }\end{array}$ \\
\hline $\begin{array}{l}\text { Depth-Based } \\
\text { Gait Dataset } \\
{[?]}\end{array}$ & 2013 & PR & 29 & $\begin{array}{c}2 \times \text { kinect } \mathrm{v} 1 \\
\text { fixed at } \\
2.5 \mathrm{~m} \text { high }\end{array}$ & $2(\mathrm{~F}+\mathrm{B})$ & $\begin{array}{c}4 \\
(2 \mathrm{~F}+2 \mathrm{~B})\end{array}$ & $\begin{array}{c}\text { VP, OC, } \\
\text { FR, WC } \\
(\mathrm{NW}+\mathrm{FW})\end{array}$ & $\mathrm{D}, \mathrm{SK}, \mathrm{T}$ & - \\
\hline $\begin{array}{l}\text { DGait } \\
5\end{array}$ & 2012 & GR & $\begin{array}{c}55 \\
360^{7}+19\end{array}$ & $\begin{array}{l}\text { kinect v1 } \\
\text { fixed at } \\
2 \mathrm{~m} \text { high }\end{array}$ & $\begin{array}{c}8(\mathrm{~F}+\mathrm{B} \\
+2 \mathrm{~S}+2 \mathrm{D} 45 \\
+2 \mathrm{D}-45) \\
\end{array}$ & $\begin{array}{c}11(2 \mathrm{~F}+\mathrm{B}+ \\
4 \mathrm{~S}+2 \mathrm{D} 45 \\
+2 \mathrm{D}-45) \\
\end{array}$ & VP & $\mathrm{C}, \mathrm{D}, \mathrm{SK}$ & $\begin{array}{c}\text { start-end } \\
\text { frame/gait } \\
\text { cycle }\end{array}$ \\
\hline $\begin{array}{l}\text { GRIDDS } \\
23\end{array}$ & 2018 & $\mid \begin{array}{c}\mathrm{PR} \\
+ \\
\mathrm{GR}\end{array}$ & $\begin{array}{c}35 \\
110^{7}+24\end{array}$ & $\begin{array}{l}\text { kinect v2 } \\
\text { fixed at } \\
1.8 \mathrm{~m} \mathrm{high}\end{array}$ & $2 \mathrm{~S}$ & $\begin{array}{c}10 \\
(5 \mathrm{~S}+5 \mathrm{~S})\end{array}$ & - & $\mid \begin{array}{c}\mathrm{C}, \mathrm{D}, \mathrm{SK} \\
\mathrm{T}, \mathrm{DS}, \mathrm{S}\end{array}$ & $\begin{array}{c}\text { start-end } \\
\text { frame/gait } \\
\text { cycle }+ \\
\text { matlab } \\
\text { scripts }\end{array}$ \\
\hline $\begin{array}{l}\text { Kinect Gait } \\
\text { Biometry } \\
\text { Dataset } \\
{[1}\end{array}$ & 2014 & PR & \begin{tabular}{|c|}
164 \\
$17-35$ \\
years old
\end{tabular} & $\begin{array}{c}2 \times \text { kinect } \mathrm{v} 1 \\
\text { in motion }\end{array}$ & $1 \mathrm{SC}$ & $5 \mathrm{SC}$ & - & SK & - \\
\hline $\begin{array}{l}\text { RGB-D } \\
\text { Person } \\
\text { Re-identifica- } \\
\text { tion Dataset } \\
2 \\
\end{array}$ & 2012 & PR & 79 & $\begin{array}{l}\text { kinect v1 } \\
\text { fixed }\end{array}$ & $2(\mathrm{~F}+\mathrm{B})$ & $\begin{array}{c}4 \\
(3 \mathrm{~F}+1 \mathrm{~B})\end{array}$ & $\begin{array}{c}\mathrm{T}, \mathrm{C} \\
\mathrm{WC} \\
(\mathrm{SW}+\mathrm{NW})\end{array}$ & $\begin{array}{l}\mathrm{C}, \mathrm{SK}, \mathrm{S}, \\
\text { 3DM, EF }\end{array}$ & $\begin{array}{l}\text { matlab } \\
\text { scripts }\end{array}$ \\
\hline $\begin{array}{l}\text { SAIVT-DGD } \\
{[25}\end{array}$ & 2011 & PR & 15 & $\begin{array}{l}\text { kinect v1 } \\
\text { fixed }\end{array}$ & $1 \mathrm{~F}$ & $20 \mathrm{~F}$ & $\begin{array}{c}\mathrm{WC} \\
(\mathrm{NW}+\mathrm{FW}), \\
\mathrm{CC} \\
(\mathrm{BC}+\mathrm{SC}+ \\
\mathrm{FC})+\mathrm{S}\end{array}$ & $\begin{array}{c}\mathrm{D}, \mathrm{DS}, \\
3 \mathrm{DV}\end{array}$ & $\begin{array}{c}\text { matlab } \\
\text { scripts }+ \\
\text { documenta- } \\
\text { tion }\end{array}$ \\
\hline $\begin{array}{l}\text { SDUgait } \\
31\end{array}$ & 2015 & PR & $\mid \begin{array}{c}52 \\
280^{7}+24\end{array}$ & $\begin{array}{c}2 \times \text { kinect v2 } \\
\text { fixed at } \\
1 \mathrm{~m} \text { high }\end{array}$ & $\begin{array}{l}\text { Kinect1: } 5 \\
\text { F+B+S+ } \\
\text { D45+A } \\
\text { Kinect2: } 5 \\
\text { F+2S+ } \\
\text { D-45+A }\end{array}$ & 5 & VP & DS, SK & $\mathrm{C} \#$ code \\
\hline $\begin{array}{l}\text { SZU RGB-D } \\
\text { Gait Dataset } \\
\text { 33 }\end{array}$ & 2013 & PR & 99 & $\begin{array}{c}\text { ASUS Xtion } \\
\text { PRO LIVE } \\
\text { fixed at } \\
0,8 \mathrm{~m} \text { high }\end{array}$ & $\begin{array}{c}4 \\
(2 \mathrm{~S}+2 \mathrm{D} 30)\end{array}$ & $\begin{array}{c}8 \\
(4 S+4 D 30)\end{array}$ & VP & $\mathrm{D}, \mathrm{DS}$ & - \\
\hline $\begin{array}{l}\text { TUM-GAID } \\
\text { Database } \\
{[16}\end{array}$ & 2012 & PR & 305 & $\begin{array}{l}\text { kinect } \mathrm{v} 1 \\
\text { fixed at } \\
1.9 \mathrm{~m} \mathrm{high}\end{array}$ & $2 \mathrm{~S}$ & $10^{5}$ & $\begin{array}{c}\mathrm{CC}, \mathrm{S} \\
\mathrm{T}^{6}, \mathrm{C}^{6}\end{array}$ & $\mathrm{C}, \mathrm{D}, \mathrm{A}$ & - \\
\hline $\begin{array}{l}\text { UPCV Gait } \\
\text { Dataset } \\
18\end{array}$ & 2013 & GR & \begin{tabular}{|c|}
30 \\
$15 \sigma^{\top}+15 q$ \\
$23-55$ \\
years old
\end{tabular} & $\begin{array}{l}\text { kinect v1 } \\
\text { fixed at } \\
2 \mathrm{~m} \text { high }\end{array}$ & $1 \mathrm{~S}$ & $5 \mathrm{~S}$ & - & SK & $\begin{array}{l}\text { matlab } \\
\text { scripts }\end{array}$ \\
\hline
\end{tabular}

1 PR: Person Recognition; GR: Gender Recognition.

2 F: Frontal; B: Backwards; S: Side (Left-to-Right and/or Right-to-Left); Dxx: Diagonal at xx Degrees; SC: Semi-Circular; MS: Moving Sensor; A: Arbitrary; IN: Irregular Number.

3 VP: Viewpoint; WC: Walking Conditions (SW: Slow Walk, NW: Normal Walk, FW: Fast Walk); C: Clothing; S: Shoes; FR: Frame Rate; OC: Occlusions; CC: Carrying Conditions (BC: Back Carrying, SC: Side Carrying, FC: Front Carrying); T: Time; SP: Sensor Position.

4 SK: 3D Skeleton Coordinates; T: Time; C: RGB data; D: Depth data; A: Audio; S: Silhouettes; 3DM: 3D Mesh; EF: Estimated Floor; 3DV: 3D Volumes; DS: Depth Silhouettes.

510 sequences for 273 subjects and 20 sequences for 32 subjects.

6 Only applied to 32 subjects. 
6. Bouchrika, I., Carter, J.N., Nixon, M.S.: Towards automated visual surveillance using gait for identity recognition and tracking across multiple non-intersecting cameras. Multimedia Tools and Applications 75(2), 1201-1221 (2016)

7. Boulgouris, N.V., Hatzinakos, D., Plataniotis, K.N.: Gait recognition: A challenging signal processing technology for biometric identification. IEEE Signal Processing Magazine 22(6), 78-90 (2005)

8. Cheng, H., Liu, Z., Zhao, Y., Ye, G.: Real world activity summary for senior home monitoring. In: Proceedings of the IEEE International Conference on Multimedia and Expo. pp. 1-4. ICME (2011)

9. Dobson, F., Morris, M.E., Baker, R., Graham, H.K.: Gait classification in children with cerebral palsy: A systematic review. Gait \& Posture 25(1), 140-152 (2007)

10. Firman, M.: RGBD datasets: Past, present and future. In: Proceedings of the IEEE Conf. on Computer Vision and Pattern Recognition - Workshops. IEEE (2016)

11. Galinska, K., Luboch, P., Kluwak, K., Bieganski, M.: A database of elementary human movements collected with RGB-d type camera. In: Proceedings of the 6th IEEE International Conference on Cognitive Infocommunications. IEEE (2015)

12. Galna, B., Barry, G., Jackson, D., Mhiripiri, D., Olivier, P., Rochester, L.: Accuracy of the microsoft kinect sensor for measuring movement in people with parkinson's disease. Gait \& Posture 39(4), 1062-1068 (2014)

13. Gouwanda, D., Senanayake, S.M.N.A.: Emerging trends of body-mounted sensors in sports and human gait analysis. In: Proceedings of the 4th International Conference on Biomedical Engineering. pp. 715-718. Springer Berlin Heidelberg (2008)

14. Gross, R., Shi, J.: The CMU motion of body (MoBo) database. Tech. Rep. CMURI-TR-01-18, Carnegie Mellon University, Pittsburgh, PA (2001)

15. Hausdorff, J.M., Rios, D.A., Edelberg, H.K.: Gait variability and fall risk in community-living older adults: A 1-year prospective study. Archives of Physical Medicine and Rehabilitation 82(8), 1050-1056 (2001)

16. Hofmann, M., Geiger, J., Bachmann, S., Schuller, B., Rigoll, G.: The TUM gait from audio, image and depth (GAID) database - multimodal recognition of subjects and traits. Journal of Visual Communication and Image Representation 25(1), 195-206 (2014)

17. Ji, X., Liu, H.: Advances in view-invariant human motion analysis: A review. IEEE Transactions on Systems, Man, and Cybernetics, Part C: Applications and Reviews 40(1), 13-24 (2010)

18. Kastaniotis, D., Theodorakopoulos, I., Economou, G., Fotopoulos, S.: Gait-based gender recognition using pose information for real time applications. In: Proceedings of the 18th International Conference on Digital Signal Processing. IEEE (2013)

19. Kwolek, B., Kepski, M.: Improving fall detection by the use of depth sensor and accelerometer. Neurocomputing 168, 637-645 (2015)

20. Moeslund, T.B., Hilton, A., Krüger, V.: A survey of advances in vision-based human motion capture and analysis. Computer Vision and Image Understanding 104(2), 90-126 (2006)

21. Nixon, M.S., Tan, T., Chellappa, R.: Human Identification Based on Gait. Springer US (2006)

22. Nunes, J.F., Moreira, P.M., Tavares, J.M.R.S.: Human motion analysis and simulation tools: A survey. In: Miranda, F., Abreu, C. (eds.) Handbook of Research on Computational Simulation and Modeling in Engineering, pp. 359-388. IGI Global, Hershey, PA, USA (2016)

23. Nunes, J.F., Moreira, P.M., Tavares, J.M.R.S.: GRIDDS - a gait recognition image and depth dataset. In: VipIMAGE 2019 / VII ECCOMAS Thematic Conference on Computational Vision and Medical Image Processing (2019) 
24. Phillips, P.J., Sarkar, S., Vega, I.R., Grother, P.J., Bowyer, K.W.: The gait identification challenge problem: Data sets and baseline algorithm. In: Proceedings of the 16th International Conference on Pattern Recognition. IEEE (2002)

25. Sivapalan, S., Chen, D., Denman, S., Sridharan, S., Fookes, C.: Gait energy volumes and frontal gait recognition using depth images. In: Proceedings of the International Joint Conference on Biometrics. pp. 1-6 (2011)

26. Stasi, S.L.D., Logerstedt, D., Gardinier, E.S., Snyder-Mackler, L.: Gait patterns differ between ACL-reconstructed athletes who pass return-to-sport criteria and those who fail. The American Journal of Sports Medicine 41(6), 1310-1318 (2013)

27. Vasconcelos, M.J.M., Tavares, J.M.R.S.: Human motion analysis: Methodologies and applications. In: Proceedings of the 8th International Symposium on Computer Methods in Biomechanics and Biomedical Engineering. CMBBE (2008)

28. Vernon, S., Paterson, K., Bower, K., McGinley, J., Miller, K., Pua, Y.H., Clark, R.A.: Quantifying individual components of the timed up and go using the kinect in people living with stroke. Neurorehabilitation and Neural Repair 29(1), 48-53 (2014)

29. Wang, J., She, M., Nahavandi, S., Kouzani, A.: A review of vision-based gait recognition methods for human identification. In: Proceedings of the International Conference on Digital Image Computing: Techniques and Applications. pp. 320327. DICTA, IEEE (2010)

30. Wang, L., Hu, W., Tan, T.: Recent developments in human motion analysis. Pattern Recognition 36(3), 585-601 (2003)

31. Wang, Y., Sun, J., Li, J., Zhao, D.: Gait recognition based on 3D skeleton joints captured by kinect. In: Proceedings of the IEEE International Conference on Image Processing. pp. 3151-3155. ICIP, IEEE (2016)

32. Webster, D., Celik, O.: Systematic review of kinect applications in elderly care and stroke rehabilitation. Journal of NeuroEngineering and Rehabilitation 11(1) (2014)

33. Yu, S., Wang, Q., Huang, Y.: A large RGB-D gait dataset and the baseline algorithm. In: Biometric Recognition, pp. 417-424. Springer International Publishing (2013)

34. Zhang, Z.: Microsoft kinect sensor and its effect. IEEE Multimedia 19(2), 4-10 (2012)

\title{
A Datasets' URLs
}

\author{
- BVH MoCap Databse \\ https://github.com/trafbarak/Database-for-gait-recognition-research- \\ - Depth-Based Gait Dataset \\ http://www.facweb.iitkgp.ac.in/ shamik/Gait/Dataset1.html \\ - DGait \\ http://www.cvc.uab.es/DGaitDB \\ - GRIDDS \\ http://gridds.ipvc.pt
}


- Kinect Gait Biometry Dataset https://bit.1y/2QbDu6v

- RGB-D Person Re-identification Dataset https://bit.ly/2HLXZU7

- SAIVT-DGD

https://research.qut.edu.au/saivt/databases/saivt-dgd-database

- SDUgait https://sites.google.com/site/sdugait

- SZU RGB-D Gait Dataset http://yushiqi.cn

- TUM-GAID Database https://www.mmk.ei.tum.de/en/misc/tum-gaid-database

- UPCV Gait dataset http://www.upcv.upatras.gr/personal/kastaniotis/datasets.html 